\title{
Susceptibility to hepatitis B infection, hepatitis B/HIV co-infections and hepatitis B immunity in HIV-positive patients starting HAART in Durban, South Africa
}

\author{
FM Chonco and S Rangiah*
}

Department of Family Medicine, University of KwaZulu-Natal, Durban, South Africa

*Corresponding author, email: rangiah@ukzn.ac.za

Background: HIV/HBV co-infection remains a global threat to HIV management despite the available effective hepatitis B vaccine and hepatitis B covering antiretroviral therapy. Many studies done in South Africa and internationally showed high prevalence of HIV/hepatitis B co-infection, which mandated routine screening for both infections before initiating HAART. Fewer studies have highlighted the prevalence of hepatitis B susceptibility in the general population starting HAART and most of them were limited to children and high-risk groups. The aim of this study was to demonstrate the extent of hepatitis B susceptibility, hepatitis B/HIV co-infections and hepatitis B immunity in general HIV-infected patients.

Method: This was a retrospective review of 1066 randomly sampled files of patients initiated on HAART between January 2012 and December 2014 at two Durban hospitals. Data collection included demographic characteristic, CD4 counts and hepatitis B serology. Data were analysed for the prevalence of hepatitis B susceptibility, HIV/HBV co-infection and hepatitis B immunity, while correlations between age, CD4 count and these three groups were demonstrated. Statistical analysis was performed using SAS version 9.3 .

Results: Total prevalence of HBV susceptibility was $69.7 \%$, HBV immunity was $26.9 \%$ and true chronic HIV/HBV co-infection was $3.4 \%$, while HBVsAg positivity accounted for $8.4 \%$ of the participants. Adults were more susceptible to HBV than children, with a median age of 36 years. Stratified for age, children were more immune $(90 \%)$ to HBV than adults.

Conclusion: This study demonstrated a significantly high number of HIV-infected persons who were susceptible to hepatitis B infection in Durban, South Africa, where both HIV and HBV are endemic, co-infection is high, and safe and effective HBV vaccine is available. Hepatitis B vaccination of the hepatitis B susceptible patients initiating HAART in South Africa is recommended to prevent further HIV/HBV co-infection.

Keywords: hepatitis B susceptibility, hepatitis B immunity, hepatitis B/ HIV co-infection, hepatitis B vaccination, HIV

\section{Introduction}

Hepatitis B Virus (HBV) and human immunodeficiency virus (HIV) co-infection remains a major health concern in sub-Saharan Africa including South Africa, ${ }^{1-6}$ where both diseases are endemic and share the same route of transmission. These studies showed a high prevalence of HBV/HIV co-infections ranging from $6 \%$ to $20 \%$ with geographic variation within the region. Two big studies done in Europe and North America showed a prevalence of $7-8 \%$ of HBV/HIV co-infection and as high as $20 \%$ prevalence was demonstrated in one province in South Africa ${ }^{6}$.

Co-infected patients have increased risk of fatal liver disease, specifically cirrhosis and death due to liver cancer. ${ }^{2,4,5,9}$ Although the current first-line highly active antiretroviral therapy (HAART) regimen covers HBV infection, drug resistance of both HIV and HBV is a threat to this treatment. ${ }^{4,10}$ Preventing HIV-infected patients from getting HBV infection therefore remains critical for the future management of HIV/HBV co-infection.

There is a safe and effective vaccine against hepatitis B infection that has been used since April 1995 in the children's Expanded Programme on Immunizations (EPI) ${ }^{9,11}$ and for non-immune medical staff in South Africa, but does not include the HBV susceptible (lack of HBV antibodies and surface antigen) HIVinfected patients. The potential delay in this regard could be due to the scarcity of local clinical data presented for clinical decision-making when interventions are planned. ${ }^{5}$ South
Africa has a vague idea of the prevalence of HBV susceptibility in the HIV- infected general population and this has potentially delayed the decision to vaccinate this susceptible group.

Some developed and developing countries that have included HBV vaccination for managing HIV-infected patients have reported good long-term public health benefits. ${ }^{12-16}$ Studies done in sub-Saharan Africa reporting on HBV have focused mainly on the prevalence of acute and chronic infections, HBV/ HIV co-infection in women or in pregnant women only, ${ }^{17,18}$ men having sex with men and children. ${ }^{11}$ The few that were inclusive of the general HIV-positive population were limited by a small sample size. ${ }^{5,6}$ Larger studies that include adults and children are needed to inform South African decision-making in the prevention of HBV/HIV co-infection.

This study aimed to determine the extent of HBV susceptibility in HIV-infected patients in Durban, KwaZulu-Natal province in South Africa. The study showed the prevalence of HBV susceptibility, HBV/HIV co-infection and immunity but did not demonstrate the incidence rate of HBV in this population.

\section{Methodology}

This retrospective, descriptive and cross-sectional study was conducted at Masibambisane and Vusithemba Antiretroviral Clinics at Wentworth and Prince Mshiyeni hospitals respectively. The study sample consisted of HIV positive patients who were 
initiated on HAART between January 1, 2012 and December 31, 2014. The pilot audit, which included 66 patients at the Masibambisane clinic showed that $47 \%$ of the patients initiating HAART at Wentworth hospital in March 2013 were susceptible to hepatitis B infection (unpublished data). A sample size of 1064 was required to estimate a proportion of $47 \%$ with a desired precision of $3 \%$ for a $95 \%$ confidence interval, with 1066 files being reviewed. To eliminate selection bias, clinical files were systematically and randomly selected, with every second file being identified until 1066 files were retrieved, half from each site. Neonates were excluded, as they were not fully vaccinated according to the South African EPI, as were pregnant women, as they were initiated on treatment in separate clinics from the general HIV-infected patients.

We reviewed the files for the routine hepatitis B serology, baseline characteristics, and included age, gender and CD4 count at the initiation of HAART. The missing data were searched using the TRAKCARE electronic system that is used to store data at both clinics. Hepatitis screening was done by the National Health Laboratory Services at Inkosi Albert Luthuli Central hospital, and no further phlebotomy was conducted to replace lost or missing data. The collected data were entered onto an approved MS Excel (Microsoft Corp, Redmond, WA, USA) spreadsheet, deidentified using a coding system and stored in a USB locked in a private cabinet to protect the patients' confidentiality.

The statistical analysis was performed using SAS version 9.3 (SAS Institute, Cary, NC) and $p$-values of $<0.05$ were considered significant. Simple mean and percentage calculations were used to analyse the baseline characteristics and the prevalence of hepatitis B susceptibility, HBV/HIV co-infection and immunity. The Wilcoxon rank sum test was used to compare differences in CD4 between adult males and females. Fisher's exact test was used to compare proportions of gender and age group across different HBVsAg categories. The Kruskal-Wallis test was used to compare $\mathrm{CD} 4$ count distribution across different HBVsAg categories

The study was approved by the University of KwaZulu-Natal Biomedical Research Ethics Committee, KwaZulu-Natal Provincial Government Department of Health and the participating antiretroviral sites in Durban, South Africa.

\section{Results}

In this review of 1066 files, the males and females were almost equally represented; there were more adults than children. An
Table 1: Baseline characteristics and CD4 count characteristics of HIVinfected patients $(n=1066)$

\begin{tabular}{llc}
\hline Variable & Level & $\boldsymbol{N}$ \\
\hline Gender, $n$ (\%) & Female & $530(49.7 \%)$ \\
& Male & $536(50.3 \%)$ \\
\hline Age group, $n(\%)$ & $<12$ years & $77(7.2 \%)$ \\
& $\geq 12$ years & $989(92.8 \%)$ \\
\hline Median CD4 count, cells $/ \mathrm{mm}^{3}$ (IQR) & All patients & $167(60-296)$ \\
\hline Median CD4 count, cells $/ \mathrm{mm}^{3}$ (IQR) & $<12$ years & $670(447-908)$ \\
\hline Median CD4 count, cells $/ \mathrm{mm}^{3}$ (IQR) & $\geq 12$ years & $151(57-269)$ \\
\hline Median CD4 count, cells $/ \mathrm{mm}^{3}$ (IQR) & Male & $132(48-245)$ \\
\hline Median CD4 count, cells $/ \mathrm{mm}^{3}$ (IQR) & Female & $171(68-291)$ \\
\hline
\end{tabular}

overall low median CD4 count of 167 cells/ml was demonstrated but, when separately analysed, children had higher CD4 counts than adults and males had a lower CD4 count median than females (Table 1).

Of the selected 1066 files, 144 had missing data, with 992 files being analysed for hepatitis B serology. A total of $69.8 \%$ of these patients were susceptible to HBV, 3.1\% were HIV/HBV co-infected, and 25\% were HBV immune. Adults (99.2\%) showed a significantly higher susceptibility to HBV than children $(0.8 \%)$, with more adults (96.8\%) being co-infected than children (3.2\%). Adults (71.8\%) appeared more immunised that children (28.2\%) but when stratified for age, children (92.1\%) were more immunised than adults. There was no significant gender difference in the adults for susceptibility, co-infection and immunity (Table 2).

The box and whiskers plot in Figure 1 shows that adults were more susceptible and more likely to be co-infected with HBV, while children were more immune to HBV, although several outliers were also demonstrated.

\section{Discussion}

The high prevalence of hepatitis B susceptible patients (69.8\%) with a higher prevalence in adults than children demonstrated in this cohort was due to lack of HBV B vaccination of people born before the inclusion of hepatitis $B$ vaccine in the South African EPI. ${ }^{9,11}$ Even though routine screening is carried out in South Africa for hepatitis B at the initiation of HAART, vaccination of the susceptible HIV-infected patient is not routine.

Table 2: HBV susceptibility, hepatitis B immunity and HIV/HBV co-infection by age, gender and CD4 count ( $n=922)$

\begin{tabular}{|c|c|c|c|c|c|}
\hline Variable & Level & $\begin{array}{c}\text { HBVsAg- and HBVsAb } \\
- \\
(n=643) \\
(=69.8 \%) \\
n(\%)\end{array}$ & $\begin{array}{c}\text { HBVsAg- and HBVsAb } \\
+ \\
(n=248) \\
(=25.0 \%) \\
n(\%)\end{array}$ & $\begin{array}{c}\text { HBVsAg+ and HBVsAb } \\
+ \\
(n=31) \\
(=3.1 \%) \\
n(\%)\end{array}$ & $p$-value \\
\hline \multirow[t]{2}{*}{ Gender } & Female & $330(51.3 \%)$ & $119(48.0 \%)$ & $14(45.2 \%)$ & $0.560^{*}$ \\
\hline & Male & $313(48.7 \%)$ & $129(52.0 \%)$ & $17(54.8 \%)$ & \\
\hline Median age, years (IQR) & & $36(30-44)$ & $30(11-42)$ & $34(30-40)$ & \\
\hline \multirow[t]{2}{*}{ Age group } & $<12$ years & $5(0.8 \%)$ & $70(28.2 \%)$ & $1(3.2 \%)$ & $<0.001^{*}$ \\
\hline & $\geq 12$ years & $638(99.2 \%)$ & $178(71.8 \%)$ & $30(96.8 \%)$ & \\
\hline Median CD4 count, cells $/ \mathrm{mm}^{3}$ (IQR) & & $145(57-260)$ & $294(124-586)$ & $123(35-307)$ & \\
\hline \multirow{2}{*}{$\begin{array}{l}\text { Median CD4 count, cells } / \mathrm{mm}^{3} \\
\text { (IQR) }\end{array}$} & $<12$ years & $322(300-488)$ & $695(450-909)$ & 113 & \\
\hline & $>-12$ years & $143(56-256)$ & $204(84-331)$ & $111(35-294)$ & $<0.001^{* *}$ \\
\hline
\end{tabular}

*Fisher's exact test was used to compare proportions of gender and age group across different HBVsAg categories

**Kruskal Wallis test was used to compare CD4 count distribution across different HBVsAg categories 


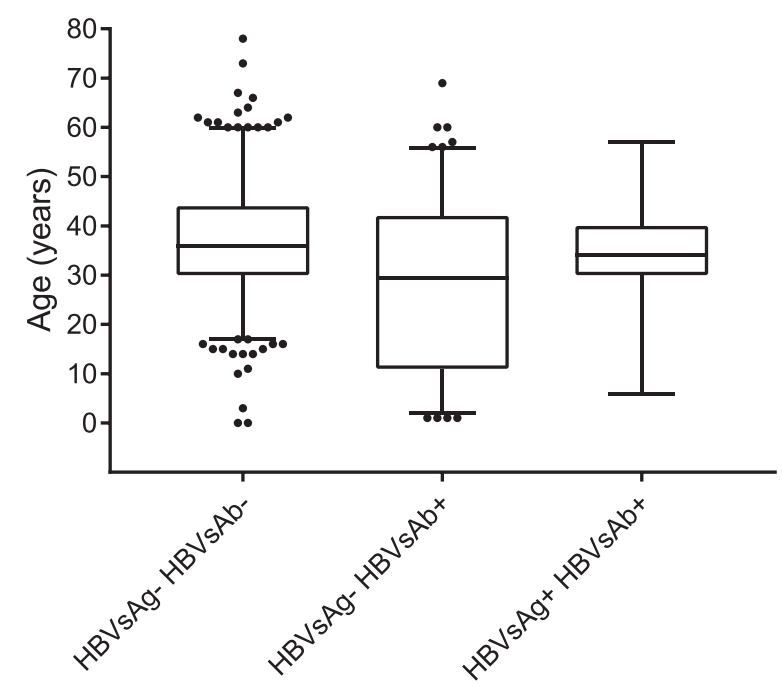

Figure 1: Age distribution across HBV groups among patients starting HAART.

The prevalence of HBV/HIV was higher (25\%) than in most studies done in South Africa. This was even higher than the recent $20 \%{ }^{6}$ reported by a study done in Limpopo province. Both HBV and HBV are endemic ( $>8 \%$ prevalence) in South Africa ${ }^{2,4}$ and they share a common route of transmission in adults, and this puts susceptible South Africans at high risk of acquiring both diseases.

Immunity was low (3.1\%) because most adults did not receive $\mathrm{HBV}$ vaccine when they were children. When comparing adults with children, immunity appeared more prevalent in adults because there were more represented than children and the data was skewed in their favour. Stratified for age, children (92.1\%) showed higher HBV immunity than adults because they were born after the inclusion of HBV immunisation for HBV.

Gender made no significant difference $(p=0.560)$ in prevalence of HBV susceptibility, co-infection and immunity because males and females of the same age were not vaccinated when born before 1995.

The study showed low CD4 at the initiation of HAART, which is a common trend with most studies done in South Africa.

\section{Conclusion}

The public health implication of the study result showed a significantly high hepatitis B susceptibility in HIV-positive patients initiating HAART in Durban, South Africa. These findings motivate for the inclusion of HBV vaccination in the management of susceptible patients initiating HAART in South Africa. The study results, supported by other similar studies, can be used to inform decision-making when planning for the prevention of HBV/HBV co-infection in South Africa. The study results will be made available to the KwaZulu-Natal and the South African Departments of Health for them to make informed clinical decisions concerning prevention of HBV/HIV co-infection. We recommend future cohort studies to explain the patterns that emerged from this cross-sectional study. This was one of the largest primary healthcare-based studies to describe hepatitis B serology amongst the general population, including females, males, adults and children, and not focusing only on pregnant women. As a retrospective study it could not explain the causes of the hepatitis B serology patterns demonstrated by the findings. Age was a confounder when analysing the data but was stratified for to account for the skewed results.

Acknowledgements - The authors would like to thank the participating sites, their staff for assisting in data collection and Ms Nonhlanhla Nyende for her statistical contribution.

Disclosure statement - There is no conflicting interest between the study and the funders.

Funding - This study was funded by the Discovery Foundation Fund No 035988, South Africa.

Authors' contribution - F.C. was responsible for the project design, data collection and presentation of results. S.R. made major contributions and corrections during the project design and writing of the manuscript.

\section{References}

1. Burnett R, François G, Kew MC, et al. hepatitis B virus and human immunodeficiency virus co-infection in sub-Saharan Africa: a call for further investigation. Liver Int 2005;25(2):201-13. http://doi.org/ 10.1111/j.1478-3231.2005.01054.x

2. Firnhaber C, Reyneke A, Schulze D, et al. The prevalence of hepatitis B co-infection in a South African urban government HIV clinic. S Afr Med J. 2008;98:541-4.

3. Mandiwana A, Tshitenge S. Prevalence of human immunodeficiency virus - hepatitis B virus co-infection amongst adult patients in Mahalapye, Ngami, Serowe, Botswana: a descriptive cross-sectional study. S Afr Fam Pract. 2017;59(3):94-7.

4. Kew M. hepatitis $B$ virus infection: the burden of disease in South Africa. South Afr J Epidemiol Infect. 2008;23(1):4-8. http://doi.org/ 10.1080/10158782.2008.11441293

5. King J, Hagemeister D. hepatitis B co-infection in HIV-infected patients receiving antiretroviral therapy at the TC Newman AntiRetroviral Treatment Clinic in Paarl, Western Cape. S Afr J HIV Med. 2016;17(1):541. http://doi.org/10.4102/sajhivmed.v17i1.336

6. Ayuk J, Mphahlele J, Bessong P. hepatitis B virus in HIV-infected patients in Northeastern South Africa: prevalence, exposure, protection and response to HAART. S Afr Med J. 2013;103(5):330-3. http:// doi.org/10.7196/SAMJ.6304

7. Konopnicki, D, Mocroft A, de Wit S, et al. hepatitis B and HIV: prevalence, AIDS progression, response to highly active antiretroviral therapy and increased mortality in the EuroSIDA cohort. AIDS. 2005;19(6):593-601. http://doi.org/10.1097/01.aids.0000163936. 99401.fe

8. Scott E, Kellerman D, Hanson A, et al. Prevalence of chronic hepatitis $B$ and incidence of acute hepatitis $B$ infection in human immunodeficiency virus-infected subjects. J Infect Dis. 2003;188(4):571-7. http://doi.org/10.1086/377135

9. Hoffmann C, Thio LC. Clinical implications of HIV and hepatitis B coinfection in Asia and Africa. Lancet Infect Dis. 2007;7(6):402-9. http:// doi.org/10.1016/S1473-3099(07)70135-4

10. Núñez $M$, Soriano V. Management of patients co-infected with hepatitis B virus and HIV. Lancet Infect Dis. 2005;5:374-82. http://doi.org/ 10.1016/S1473-3099(05)70141-9

11. Jooste $P$, van Zyl A, Adland $E$, et al. Screening, characterisation and prevention of hepatitis B virus (HBV) co-infection in HIV-positive children in South Africa. J Clin Virol. 2016;85:71-4. http://doi.org/10. 1016/j.jcv.2016.10.017

12. Abara W, Qaseem A, Schillie S, et al. hepatitis B vaccination, screening, and linkage to care: best practice advice from the American College of Physicians and the Centers for Disease Control and Prevention. Ann Intern Med. 2017;167(11):794-804. http://doi.org/ 10.7326/M17-1106

13. Martins $S$, do Livramento Ad, Andrigueti $M$, et al. Vaccination coverage and immunity against hepatitis $B$ among HIV-infected patients in South Brazil. Braz J Infect Dis. 2015;19(2):181-6. http://doi.org/10. 1016/j.bjid.2014.12.002 
14. Tedaldi EM, Baker RK, Moorman AC, et al. hepatitis A and B vaccination practices for ambulatory patients infected with HIV. Clin Infect Dis. 2004;38(10):1478-84. http://doi.org/10.1086/420740

15. Phung BC, Launay $O$. Vaccination against viral hepatitis of HIV-1 infected patients. Hum Vaccin Immunother. 2012;8(5):554-9. http:// doi.org/10.4161/hv.19105

16. Tithiah N, Parboosing $R$, Singh $L$, et al. Human immunodeficiency virus and hepatitis $B$ or C co-infection in KwaZulu- Natal: a retrospective analysis of a laboratory database.
17. Coffie PA, Egger M, Vinikoor MJ, et al. Trends in hepatitis B virus testing practices and management in HIV clinics across subSaharan Africa. BMC Infect Dis. 2017;17(Suppl 1):706. http://doi.org/ 10.1186/s12879-017-2768-z

18. Chambal LM, Gudo ES, Carimo A, et al. HBV infection in untreated HIV-infected adults in Maputo, Mozambique. PLOS ONE. 2017;12(7): e0181836. http://doi.org/10.1371/journal.pone.0181836

Received: 28-05-2018 Accepted: 23-08-2018 